\title{
Open Issues and Opportunities in Web Services Modeling, Development, and Management
}

\author{
Fabio Casati \\ Hewlett-Packard \\ 1501 Page Mill road, MS 1142 \\ Palo Alto, CA, 94304, USA \\ fabio. casati@hp.com
}

\section{Extended Abstract}

Despite the great interest and the enormous potential, Web services, and more in general service-oriented architectures (SOAs), are still in their infancy. The speed at which software vendors have released middleware and development tools for Web services is unprecedented, but their level of maturity is still far from their counterparts in conventional middleware. This is only natural and common to any novel technology, and will be corrected over time, as more users adopt this technology and provide feedback on what the actual needs are.

Aside from considerations related to the young age of the technology, the interesting aspect of Web services is that they have characteristics that differ from services in conventional middleware, and that can service development and management tools to a new dimension. For example, services in conventional middleware were often tightly coupled and developed by the same team. This means that it was difficult to combine services in different ways, and that service descriptions were rather poor. Web services are instead loosely-coupled and designed for B2B applications. This means that Web services are independent of each other and are invoked by clients who have no access to the service development team. Since service descriptions are all clients have available to understand the service behavior, they are richer than their counterpart in conventional middleware, going well beyond interface descriptions. This factor, along with the increased standardization and the loose coupling, makes composition technologies more applicable. The opportunity of composing Web services and of leveraging richer service descriptions are some of the factors that both create more requirements and enable a greater level of automated support from the middleware and from CASE-like development tools.

Other important aspects are related not so much to Web service technology, but to their intended applicability. For example, Web services are typically used to integrate complex systems and not to perform simple computations. Hence, manageability and ease of deployment become often more important than performance.

In this presentation I will elaborate on these and other key aspects of Web services, emphasizing the opportunities they provide and the unsolved challenges they present from a modeling and management perspective. 